Corrected: Publisher correction

ARTICLE

DOI: $10.1038 / \mathrm{s} 41467-018-06502-9$

\title{
Complex virome in feces from Amerindian children in isolated Amazonian villages
}

\author{
Juliana D. Siqueira (10) ${ }^{1,2}$, Maria Gloria Dominguez-Bello ${ }^{3}$, Monica Contreras ${ }^{4}$, Orlana Lander ${ }^{5}$, \\ Hortensia Caballero-Arias ${ }^{6}$, Deng Xutao ${ }^{1,7}$, Oscar Noya-Alarcon ${ }^{5,8}$ \& Eric Delwart ${ }^{1,7}$
}

The number of viruses circulating in small isolated human populations may be reduced by viral extinctions and rare introductions. Here we used viral metagenomics to characterize the eukaryotic virome in feces from healthy children from a large urban center and from three Amerindian villages with minimal outside contact. Numerous human enteric viruses, mainly from the Picornaviridae and Caliciviridae families, were sequenced from each of the sites. Multiple children from the same villages shed closely related viruses reflecting frequent transmission clusters. Feces of isolated villagers also contained multiple viral genomes of unknown cellular origin from the Picornavirales order and CRESS-DNA group and higher levels of nematode and protozoan DNA. Despite cultural and geographic isolation, the diversity of enteric human viruses was therefore not reduced in these Amazonian villages. Frequent viral introductions and/or increased susceptibility to enteric infections may account for the complex fecal virome of Amerindian children in isolated villages.

\footnotetext{
${ }^{1}$ Blood Systems Research Institute, San Francisco, CA 94118, USA. ${ }^{2}$ Programa de Oncovirologia, Instituto Nacional de Câncer, Rio de Janeiro 20.231-050, Brazil. ${ }^{3}$ Department of Biochemistry and Microbiology and of Anthropology, Rutgers University, New Brunswick, NJ 08901-8554, USA. ${ }^{4}$ Center for Biophysics and Biochemistry, Venezuelan Institute of Scientific Research (IVIC), Caracas 01204, Venezuela. ${ }^{5}$ Instituto de Medicina Tropical, Universidad Central de Venezuela, Caracas 1051, Venezuela. ${ }^{6}$ Department of Anthropology, Venezuelan Institute of Scientific Research (IVIC), Caracas 01204, Venezuela. ${ }^{7}$ Department of Laboratory Medicine, University of California at San Francisco, San Francisco, CA 94118, USA. ${ }^{8}$ Amazonic Center for Research and Control of Tropical Diseases (CAICET), Puerto Ayacucho 7101, Venezuela. Correspondence and requests for materials should be addressed to E.D. (email: delwarte@medicine.ucsf.edu)
} 
$\mathrm{H}$ umans in the pre-agricultural area are believed to have been widely dispersed in small nomadic groups minimizing the spread and maintenance of infectious diseases. The peopling of the American continent is believed to have occurred between $15-20 \mathrm{~K}_{\text {years ago }}{ }^{1-3}$. Amerindian populations settled Monte Verde near the southern tip of the continent at least $13 \mathrm{~K}$ years ago ${ }^{4}$ and possibly as long as $19 \mathrm{~K} \mathrm{ago}^{5}$. The founding native American population is estimated to have had a small effective population size ${ }^{6}$ and migration from the Bering strait may have occurred in one major or multiple waves ${ }^{7-10}$. Serological and PCR tests have shown that chronic viral infections such as Human T-lymphotropic Virus 2 (HTLV2), JC polyomavirus (JCV), and Human herpesvirus 8 (HHV8), may have been introduced during the continent's initial human colonization, while other subtypes of HTLV2 and JCV were likely introduced later during the slave trade into Brazil ${ }^{11-13}$. Coincident with mixing of Amerindian and later immigrant populations in urban settlements, native populations underwent strong population bottlenecks believed to be coincident with imported airborne epidemics such as small pox, measles, and more recently influenza, to which they may have been particularly susceptible due to lack of prior exposure ${ }^{14,15}$.

Small populations now settled in hard to explore regions such as the rain forests in the South American Amazon and Orinoco rivers' basins may therefore still be isolated from frequent exposures to highly prevalent circulating viruses. Such small populations may also be expected to lose viruses that do not typically establish chronic or long lasting infections such as enteric or respiratory infections whose maintenance are believed to be dependent on large population size of young, seronegative, susceptible hosts found in larger populations ${ }^{16}$. A strong population bottleneck in a non-human primate species was also shown to reduce the diversity of their blood-borne viruses ${ }^{17}$.

Here we tested whether relatively isolated Amerindians populations in the Amazon showed a reduction in the diversity of their mammalian enteric viruses. We analyzed and compared the fecal virome of Amerindian children along a gradient of population size and isolation ranging from a large urban center to an isolated but exposed rainforest village that has a landing strip and two even more remote villages.

\section{Results}

Populations analyzed. Stool samples were collected from 20 children each from four locations within Venezuela. The characteristics from the villages and the samples studied are shown in Table 1. Children from urban site A (city of Caracas) had an average age of 4 years and were mestizos. The children analyzed from villages $\mathrm{B}, \mathrm{C}$, and $\mathrm{D}$ had an average age of 3,4 , and 4 years, respectively, and were Amerindian children living in communities inaccessible by land located in the South of Venezuela (Methods and Supplementary Figure 1-5). All three villages

\begin{tabular}{|c|c|c|c|c|}
\hline Location & Caracas & Kanarakuni & Surukuma & Chajuraña \\
\hline Name site used here & A & B & C & D \\
\hline $\begin{array}{l}\text { Number feces } \\
\text { analyzed }\end{array}$ & 20 & 20 & 20 & 20 \\
\hline Ethnic group & Mestizo & Yekwana & Sanema & Sanema \\
\hline $\begin{array}{l}\text { Level of } \\
\text { transculturation }\end{array}$ & High & Medium & Low/non & Low/non \\
\hline Male (gender) & $9(45 \%)$ & $11(55 \%)$ & $5(25 \%)$ & $9(45 \%)$ \\
\hline Average age (year) & 4 & 3 & 4 & 4 \\
\hline
\end{tabular}

(groups B, C, D) consume food from their gardens, hunting, and fishing, with no market food. All inhabitants from these villages speak their native language and no Spanish, except for about 5\% of the persons in site $B$.

Viral sequences recovered. Viral particle associated nucleic acids were enriched from fecal supernatant using filtration and nuclease digestion prior to nucleic acid extraction and preparation of sequencing libraries for each individual fecal sample. A total of $59,922,846$ sequence reads were generated using the MiSeq platform. Following de novo assembly using the Ensemble program both singlets and contigs were analyzed using BLASTx for translated sequence protein similarity to all the viral proteins in GenBank's virus RefSeq. Sequences from 56 viral families were identified. Considering only the 23 viral families known to infect vertebrates, the greatest number of hits belonged to the families Picornaviridae (1,552,825 sequence hits), Caliciviridae $(123,766)$, Circoviridae (44,277), Anelloviridae (28,296), Astroviridae $(12,990)$, and Reoviridae (3437). Other viral families were also detected with fewer read numbers. Over a hundred near-complete or partial genomes contigs were assembled from different viral families (Supplementary Data 1).

Viral read distribution. The number of short reads aligning to the near complete genomes/contigs generated by de novo assembly was calculated. When multiple genomes showed a high degree of nucleotide identity of $>95 \%$ the longest sequence was selected as the representative genome (Supplementary Data 1). When long viral contigs could not be generated the closest available genome sequence from GenBank was used $(12 \%$ of viruses; Fig. 1). The percentage of quality-filtered reads that aligned to the selected viral genomes/contigs selected ranged from 0.02 to $35.5 \%$ per sample.

The diversity of the eukaryotic viral genomes and the predominance of RNA viruses (particularly picornaviruses) is shown (Fig. 1). We also detected multiple children from the same locations, particularly villages $\mathrm{B}, \mathrm{C}$, and $\mathrm{D}$, infected with the same viral species or genotypes seen as sets of adjoining bubbles of the same color (Fig. 1). A total of 48 clearly human viral species or genotypes (excluding the polioviruses and rotavirus vaccine strains described below, dietary gyroviruses, unclassified CRESSDNA viruses, and unclassified Picornavirales) were identified whose distribution among the four sites varied greatly (Fig. 1). The urban children showed a lower number of enteric human virus infections (average of 0.95 ; range $0-2$ viruses) than the three villages (average of 2.70-2.75; range $0-9$ viruses). The difference in the number of human viruses per child in site A versus village $\mathrm{B}, \mathrm{C}$ and $\mathrm{D}$ was significant ( $p$ values of $0.022,0.011$, and 0.028 , respectively, using Tukey's range test). No statistically significant differences were observed between the three villages. The numbers of distinct human viruses from each of the four locations were also counted (counting the closely related variants in transmission cluster from the same location only once). The 20 urban children sampled harbored 11 distinct human viruses (excluding the vaccine derived poliovirus $1 / 2 / 3$ and rotavirus), while those from villages $\mathrm{B}, \mathrm{C}$, and $\mathrm{D}$ showed the presence of 23 , 19, and 18 distinct human viruses, respectively. When the proportion of all 48 human viruses detected in each of the four sites were compared using Chi square analysis only urban site A versus village $B$ differed significantly $(p=0.010)$. However, when we analyzed the proportion of the 33 viruses of unknown origin, the urban area site A showed a significant lower number of viruses detected (four viruses) compared to 13,13 , and 19 viruses found in villages B $(p=0.011), C(p=0.011)$ and $\mathrm{D}(p=0.0001)$, 


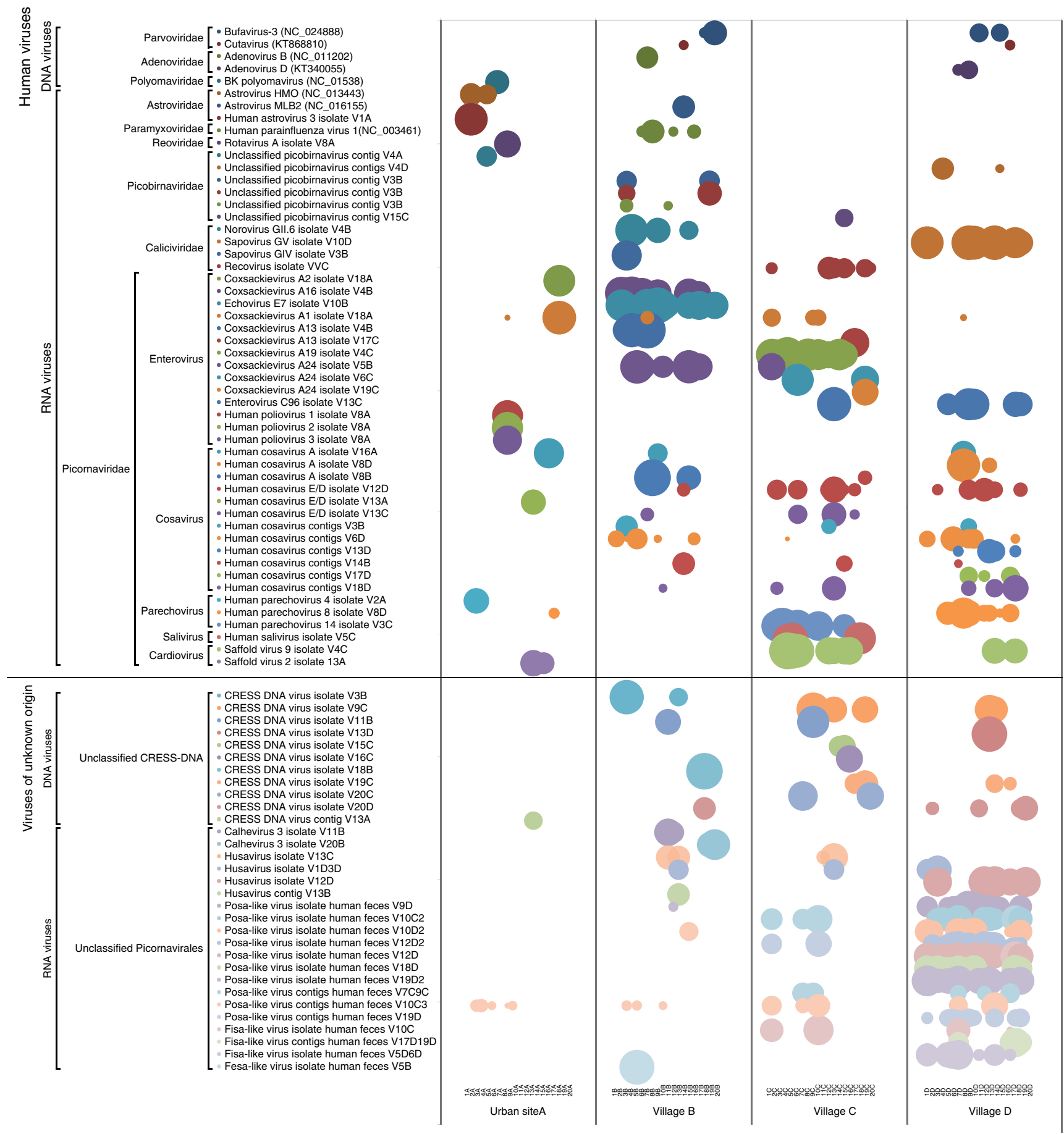

Fig. 1 Virus distribution by children in four sites. Each circle represents the log 10 transformed number of virusgenome/contig matching viral reads per million total reads

respectively. We then analyzed in details the phylogenetic relationships between genomes from the same viral families.

Picornaviridae. This ssRNA viral family was represented by 57 genomes from five genera (enterovirus, parechovirus, cardiovirus, cosavirus, and salivirus) that could be assembled from the samples yielding the largest number of viral reads (Supplementary Data 1, Fig. 2, with the location sampled indicated by color of strain name). The most commonly detected picornaviruses were enteroviruses with 32 genomes. Some enterovirus genotypes were detected only once in a given location, while for other genotypes two or more closely related variants were shed by multiple individuals from the same location. These viral phylogenetic clusters are indicative of close epidemiological linkage between infected individuals within very small communities (villages $\mathrm{B}, \mathrm{C}$, and D). For example echovirus E7 was recovered from seven children from village B. These echovirus E7 genomes were closely related ( $>99.7 \%$ nucleotide identity) (Fig. 2). Seven children from village $\mathrm{C}$ also shed nearly identical Coxsackie A19 genotype variants (>99.8\% nucleotide identity) (Fig. 2). Coxsackievirus A13 genomes found in three children from village B similarly shared high nucleotide similarity $(>99.4 \%$ ) while a fourth coxsackievirus A13 from another village (C) showed only 91\% identity. Two 


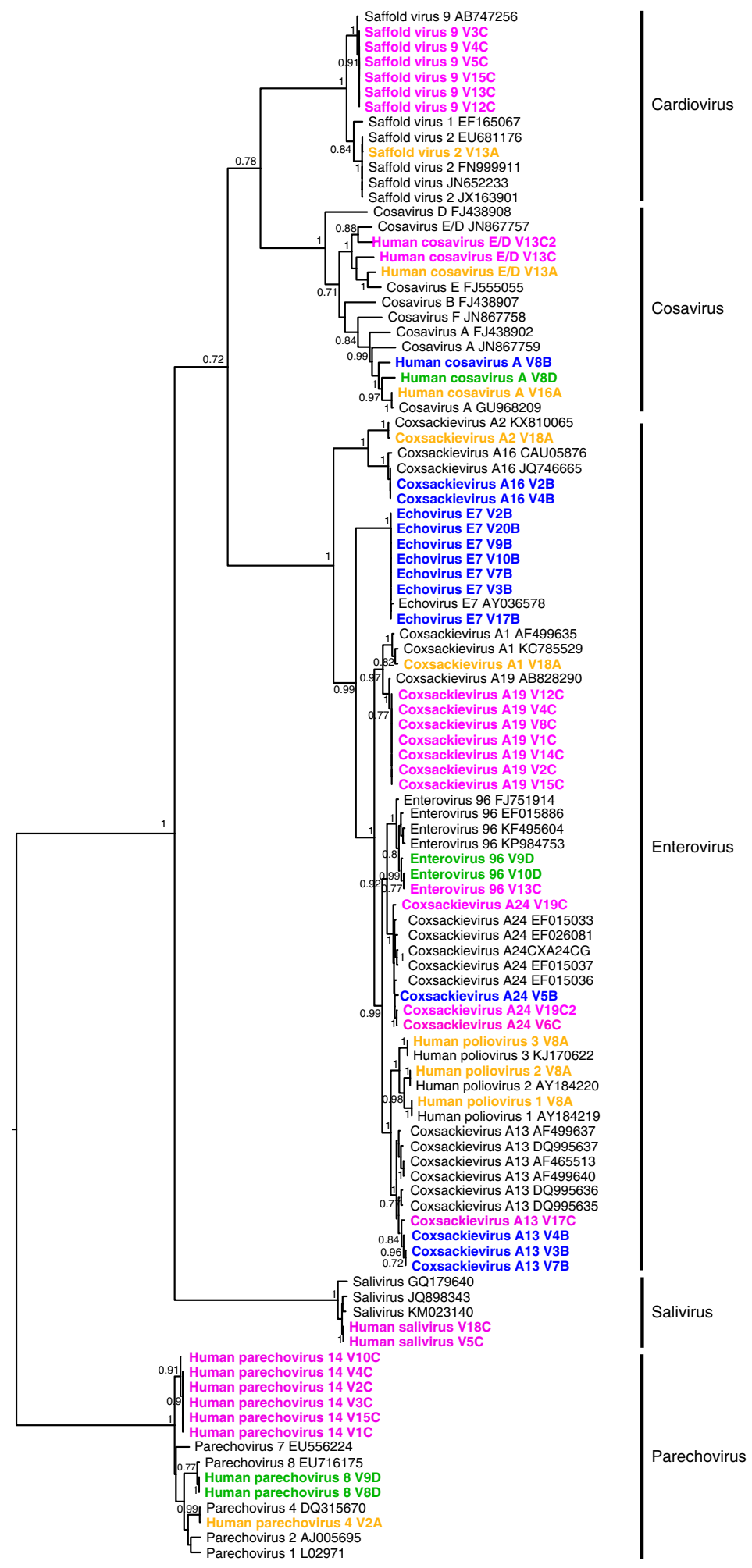

1.0

Fig. 2 Phylogenetic analysis of P1 amino acid sequences from members of the family Picornaviridae. The analysis was carried with maximum likelihood method and 100 bootstrap replicates. The viral sequences assembled in this study for which the P1 region was available are in bold and colored according to the location (Urban center $A$ in yellow; village $B$ in blue; village $C$ in magenta; and village $D$ in green). Bootstrap values higher than 0.7 (70\%) are shown. Site of sample collection is also reflected in the taxa name (containing A, B, C, or D) for each specific location. GenBank accession numbers for study sequences are listed in Supplementary Data 1 
nearly identical coxsackie A16 were also found in village B (99.97\% similarity). A more complex situation was seen with four Coxsackievirus A24 variants recovered from three children. The co-infecting Coxsackievirus A24 genomes in child 19C (from village C) shared $91 \%$ nucleotide similarity, while a genome from village $\mathrm{B}$ showed $<89.1 \%$ identity to the other genomes. The remaining coxsackie A24 from a second village C child (6C) was $99.2 \%$ identical to one of the two variants from child 19C. Two enterovirus $\mathrm{C} 96$ genomes from two village $\mathrm{D}$ children and a third from village C sample showed $>98.0 \%$ nucleotide similarity, and was the only case where picornaviruses from different villages shared a high level of similarity.

Parechovirus genomes were assembled from three sites (A, C, D) each presenting different parechovirus types (Supplementary Data 1, Fig. 2). Village C yielded six parechovirus genomes from six children sharing more than $99.95 \%$ nucleotide identity. Their VP1 regions showed the highest identity ( $94.7 \%$ amino acid) to the prototype sequence from parechovirus type 14 (FJ373179) from Amsterdam with only partial VP1 (624bp) available ${ }^{18}$. Sequences from Ghanaian children recently also classified as parechovirus type 14 (KY931642, KY931644, KY931646, and KY931647) were the next closest sequences found by BLASTn ${ }^{19}$. The six closely related village $C$ parechoviruses described here were therefore classified as type 14. Two parechovirus type 8 from village D shared $95.1 \%$ nucleotide identity. A single parechovirus type 4 was found in urban center $A$.

Seven Cardiovirus $B$ were assembled: one Saffold virus type 2 genome from urban center A, and six Saffold virus type 9 from six children in village C (Supplementary Data 1, Fig. 2). The type 9 Saffold viruses genome sequences were $>99.1 \%$ identical.

Cosavirus genomes were found in all four locations. From the seven genomes recovered three were classified as species A genotypes: genotypes A6 (urban center A), A7 (village D), and A9 (village $B)^{20}$. The other four genomes from three children (child 13C was co-infected) from locations $\mathrm{A}, \mathrm{C}$, and D were closest in their $\mathrm{P} 1$ region to the cosavirus $\mathrm{E}(\mathrm{FJ} 555055)^{21}$ (V13A and $\mathrm{V} 13 \mathrm{C}$ ), and E/D recombinant (V12D and V13C2) (Supplementary Data 1, Fig. 2). Unlike members of the other Picornaviridae genera none of the cosaviruses showed close sequence similarity.

Saliviruses were detected from two children from village $\mathrm{C}$ (V5C and V18C) showing 100\% VP1 amino acid identity. Their closest relatives were found in sewage from Bangkok, Thailand (JQ898343 with 97\% nucleotide identity) $)^{22}$ and China (KM023140 with $96 \%$ nucleotide identity ${ }^{23}$.

Contrary to the situation in the isolated villages no phylogenetic clusters with closely related picornaviruses were identified from the large urban center (site A). One child from site A shed all three Sabin poliovirus genotypes with contigs length of 73187395 nucleotides. A PV1 contig was $100 \%$ identical to Sabin 1 AY184219, a PV2 contig was $100 \%$ identical to Sabin 2 AY184220, and a PV3 contig showed a single mismatch to Sabin 3 KJ170622 (Supplementary Data 1, Fig. 2). Detection of poliovirus sequences reflects oral immunization taking place in Venezuela in 2015.

Caliciviridae. Ten genomes from this ssRNA viral family could be assembled and classified (Supplementary Data 1, Fig. 3). One norovirus GII6 and one sapovirus genotype GIV were found in village B. Seven sapovirus genomes from genotype GV were assembled from seven children in village $\mathrm{D}$ with $>99.5 \%$ pair-wise nucleotide similarity among them, indicating another phylogenetic cluster in an isolated village. From a village $C$ child, we assembled the complete main ORF of a calicivirus (MG571787) with $86.3,73.1$, and $62.4 \%$ amino acid identity in the polyprotein, VP1 and VP2, respectively, to recovirus Bangladesh/289/2007
(JQ745645) a virus initially identified in captive rhesus macaques and human diarrhea samples from Bangladesh ${ }^{24,25}$.

Other human viruses. From the ssRNA Astroviridae viral family a near complete mamastrovirus 1 genome was assembled from urban center A that was $96.9 \%$ identical to human astrovirus type 3 (HAstV-3 - AF117209). Also from site A we identified reads from the astrovirus HMO-A (aka mamastrovirus 8) in two children, the only human virus found in multiple individuals in site A. From site B a third astrovirus (MLB2) was identified. From the family Reoviridae, eleven rotavirus A (RVA) segments ranging in length from 631 to 2989 bp were generated from an urban center A child who was also shedding poliovirus $1 / 2 / 3$. Complete coding regions of seven segments were assembled and virus was classified as genotype $\mathrm{G} 1 \mathrm{P}(8)$. The highest matches for each fragment with 99-100\% nucleotide identities was with the live Rotarix vaccine strain RVA/Human-lab/USA/Rotarix_SSCRTV_00092/2016/G1P (X) (MF469214-MF469224), indicating that this child was likely to have been recently orally vaccinated with both live attenuated poliovirus $1,2,3$ and Rotarix.

From the family Paramyxoviridae reads of human parainfluenza 1 virus were detected in two children from village B. From the family Parvoviridae, genus protoparvovirus, both bufavirus 3 and cutavirus were detected in children from villages B and D. In the family Polyomaviridae we detected polyomavirus BK in one child from urban center A. Numerous reads from the family Picobirnaviridae were identified in samples from all four sites (Supplementary Data 1).

Animal viruses. From large urban center A we also detected reads from chicken anemia virus (CAV) and gyrovirus 4 , the first a known and the second a likely avian virus ${ }^{26}$, in the family Anelloviridae, genus Gyrovirus ${ }^{27}$. One sample contained both viruses, another only CAV and a third only Gyrovirus 4, presumably from dietary sources.

Picornavirales genomes of unknown origin. A total of 30 near complete genomes from ssRNA viral genomes of unknown cellular origins were assembled mostly from villages $\mathrm{C}$ and $\mathrm{D}$ (Supplementary Data 1, Fig. 4). Village D, the geographically and culturally most isolated village showed the greatest number of such Picornavirales reads. Twenty-seven genomes from villages $\mathrm{C}$ and $\mathrm{D}$ exclusively were related to posaviruses, fisavirus, and husaviruses. These approximately $10 \mathrm{~Kb}$ genomes encode a single picornavirus-like polyprotein. Phylogenetic analysis of their RNA dependent RNA polymerase (RdRp) region clustered these sequences with those from fecal samples from various mammals, including humans, in the Picornavirales order (Fig. 4).

Multiple children from village D shed closely related posaviruses (Fig. 4) as reported above for picornaviruses in village $\mathrm{C}$ and $\mathrm{D}$ and caliciviruses in village $\mathrm{D}$. Four phylogenetic clusters of 4 (V1D, V13D, V17D, and V19D2 with $>99.7 \%$ nucleotide identity), 4 (V10D, V12D, V18D3, and V19D with > 99.8\% nucleotide identity), 2 (V9D and V10D4 with 100\% nucleotide identity) and 2 posaviruses (V10D3 and V18D with > 99\% nucleotide identity) were identified in village D. Three closely related posaviruses from both villages C and D (V18D2, V12D3, and V10C2) were 99\% identical at the nucleotide level.

Clustering was also seen with husavirus genomes. Five husaviruses from village D (strains V3D, V12D, V14D, V17D, and V20D) showed $>99.8 \%$ nucleotide identity (Supplementary Data 1, Fig. 4). Two other husavirus sequences, one from village $\mathrm{D}$ (V1D3D) and other from village C (V13C), shared $98.6 \%$ nucleotide identity. The three genomes related to fisavirus 1 , two 


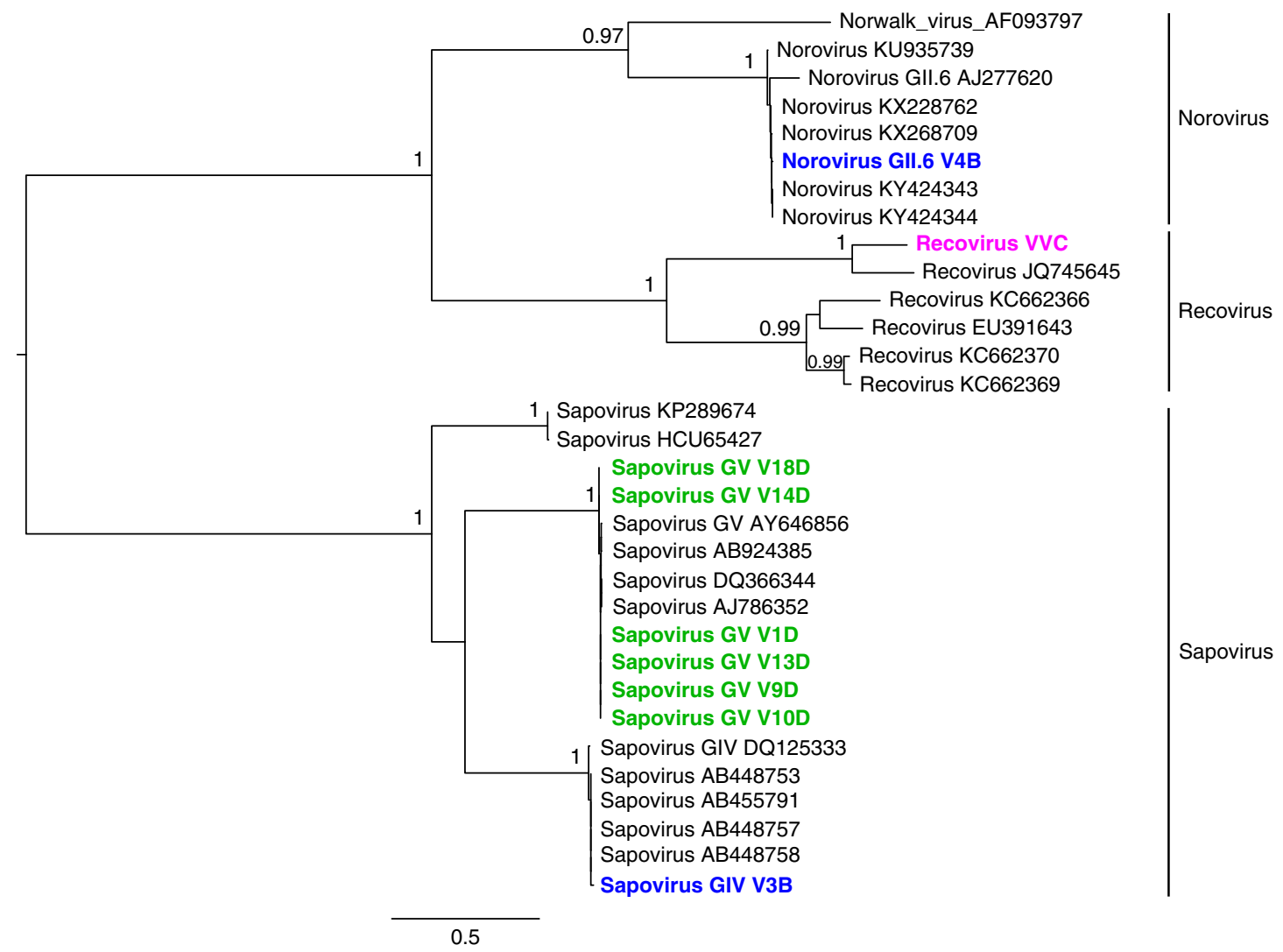

Fig. 3 Phylogenetic analysis of P1 amino acid sequences from members of the family Caliciviridae. The analysis was carried with maximum likelihood method and 1000 bootstrap replicates. The viral sequences assembled in this study for which the P1 region was available are in bold and colored according to the location (village B in blue; village $C$ in magenta; and village $D$ in green). Bootstrap values higher than 0.7 (70\%) are shown. Site of sample collection is also reflected in the taxa name (containing A, B, C, or D) for each specific location. GenBank accession numbers for study sequences are listed in Supplementary Data 1

from village $\mathrm{D}$ and one from village $\mathrm{C}$, shared $>96.4 \%$ genome identity.

Two calhevirus genomes from children in village B (V11B and V20B) were also identified. Calhevirus genomes originally identified in feces from South Asia have ORFs and nucleotide compositions reminiscent of dicistroviruses ${ }^{28-30}$. The genomes showed $95.2 \%$ nucleotide identity between them and less than $74.3 \%$ nucleotide identity to previously sequenced calheviruses (AB937990, AB937991, HM480374, and KJ950908 and KJ950908). Given that the nucleotide identity between calhevirus 1 and 2 genomes was $\sim 73 \%$, we annotated this genome as encoding calhevirus 3 .

A genome assembled from one village B (V5B) sample was also closely related to fesavirus 1 (KM017736) described in feline fecal specimens $^{31}$, and a bat felisavirus sequenced from Eidolon helvum feces ${ }^{32}$.

Circular Rep-encoding small ssDNA (CRESS-DNA) genomes of unknown origins. CRESS-DNA genomes were predominantly found in the isolated villages. A total of 16 CRESS-DNA virus contigs could be assembled (Supplementary Data 1), only one originating from urban site A. The contigs ranged in size from 1634 to 3646 bases six of which with complete circular genomes (identified with direct repeats at their extremities). Villages $\mathrm{C}$ and $\mathrm{D}$ showed cases of multiple children (two to four) excreting the same CRESS-DNA genomes (Fig. 1). For example from village C the related genomes from different children shared $>99 \%$ nucleotide identity. Several similarly closely related genomes were also found in different villages (Fig. 1).
Detection of protozoan and nematode DNA. To further investigated the presence of enteric parasites that might serve as potential cellular hosts for the unclassified RNA viruses in the Picornavirales order and CRESS-DNA genomes, we investigated the presence of eukaryotic parasites. The numbers of sequence reads from nine common eukaryotic parasites were quantified using the same method (Bowtie 2) used for the viral contigs. The intestinal nematode genome sequences used were from: Ascaris suum (large roundworm of pigs), Ascaris lumbricoides (large roundworm of human), Ancylostoma duodenale (old world hookworm), and Necator americanicus (new world hookworm). Available protozoan genomes sequences used were from: Giardia intestinalis, Entamoeba histolytica, Entamoeba dispar, Cryptosporidium hominis, and Cryptosporidium parvum. The distributions of normalized read matches were then compared between the urban center site $A$ and each village separately and combined (sites B, C, D) (Supplementary Figure 6) for all nine parasites. Statistical analysis of read numbers adjusted for multiple comparisons showed Ascaris suum, Cryptosporidium hominis, and Cryptosporidium parvum, to be significantly higher in all the villages than in the city (Supplementary Table 1). Giardia intestinalis and Ascaris lumbricoides reads were also detected in higher numbers in villages B and D relative to urban center A. Greater numbers of parasitic genome DNA reads were therefore observed in the feces of villagers relative to the urban children.

\section{Discussion}

The virome of individuals from populations of widely different size with different diet and lifestyles have rarely been contrasted 


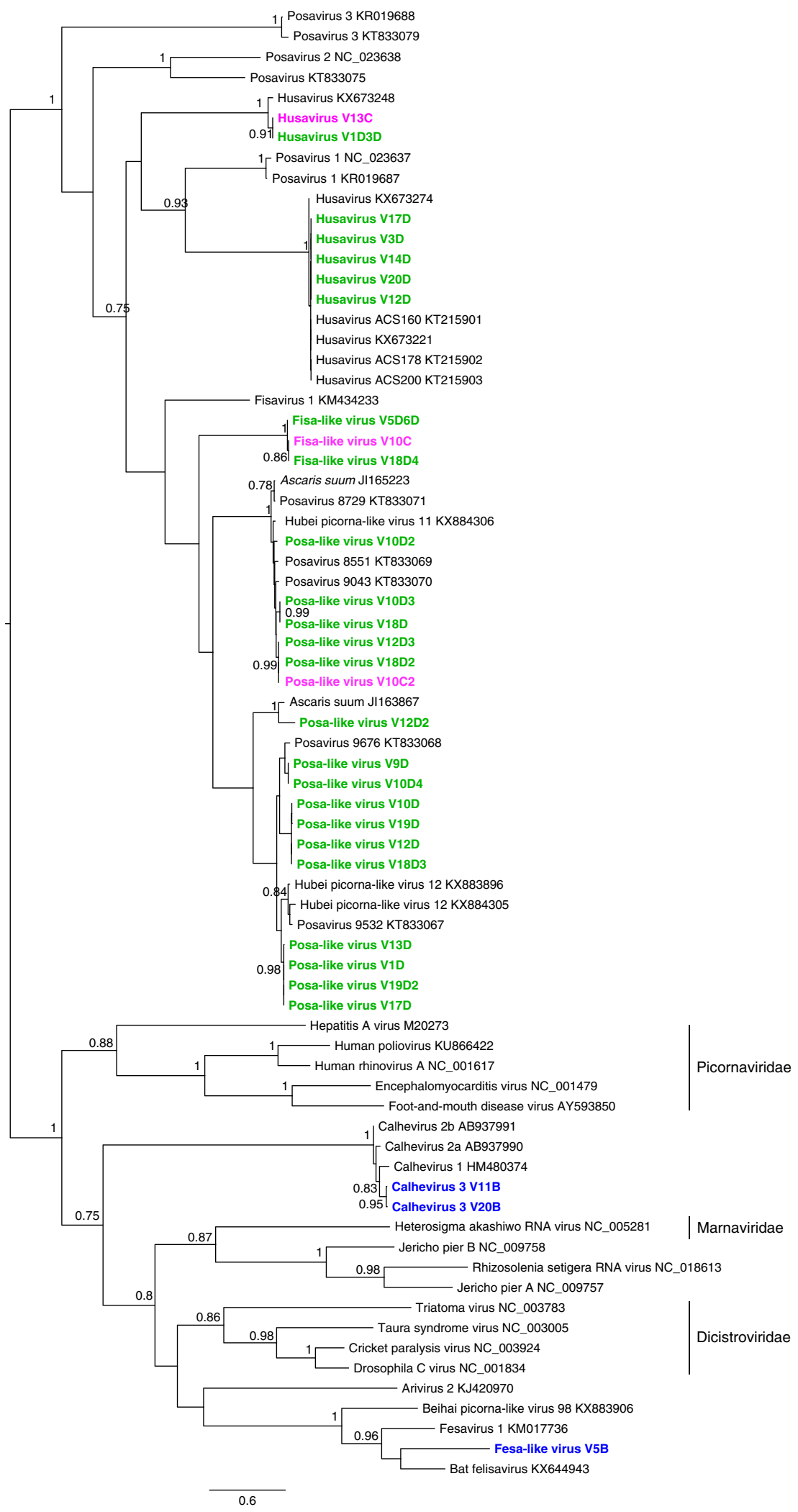

Fig. 4 Phylogenetic analysis of 459 amino acid region of RdRp from unassigned members of the Picornavirales order. The analysis was carried with maximum likelihood method and 1000 bootstrap replicates. The viruses sequences assembled in this study for which the RdRp region was available are in bold and colored according to the location (village B in blue; village $C$ in magenta; and village $D$ in green). Only bootstrap values higher than 0.7 (70\%) are shown. Site of sample collection is also reflected in the taxa name (containing A, B, C, or D) for each specific location. GenBank accession numbers for study sequences are listed in Supplementary Data 1 
using comparable methodologies. A prior study by Holtz et al. ${ }^{33}$ compared fecal samples from diarrheic infants in Melbourne and Northern Territories communities in Australia and binned reads into viral families and genera. This study reported the detection of the families Picornaviridae, Reoviridae, Caliciviridae, Adenoviridae, and Anelloviridae, all of which plus Paramyxoviridae, Picobirnaviridae, Parvoviridae, and Polyomaviridae were also found here in Venezuela. A higher number of viral families per fecal samples were reported from the Northern Territories relative to Melbourne due particularly to more adenovirus and enterovirus infections. Environmental and socioeconomic conditions, together with the generally worse health status of Northern Territories residents, were invoked as possible factors accounting for their greater rate of enteric infections. We compared here the enteric virome of age-matched healthy young infants living in communities with widely different levels of transculturation: a large urban center and three small villages in the Venezuelan Amazon with increasing levels of isolation (Supplementary Figures 1-5 and Methods). We focused on the highly diverse set of eukaryotic (particularly human) viral genomes to measure the impact of geographic isolation on human viruses believed to be dependent on large populations for their maintenance. While RNA phages are relatively sparse relative to DNA phage in human feces, the situation is generally reversed for mammalian viruses largely due to common picornavirus and calicivirus infections. The dominance of RNA viruses seen here is therefore fairly typical for human enteric virus infections ${ }^{33-39}$. The assembly of long viral genome contigs also allowed detailed phylogenetic analyses to reveal the relationships between members of the same viral species and genotype.

Enteric human viruses and other eukaryotic viruses of unknown origins were detected in feces from all four sampled sites. The number of distinct human enteric viruses in each site ranged from 11 in the urban center to 23 in village $B$. The greatest numbers of infections were by picornaviruses, predominantly from the enterovirus and cosavirus genera as well as parechoviruses, cardioviruses, and saliviruses. Multiple children were shedding nearly identical picornaviruses in the three villages relative to the infections seen in the large urban center. Several non-mutually exclusive explanations may account for this observation. These viral transmission clusters may be due to the higher probability of sampling epidemiologically linked individuals in very small villages, where most people have familial ties, than from randomly selected individuals from much larger communities. Rapid viral spread due to less sanitary conditions may also account for the high infection rates with closely related viruses within these isolated communities. The higher degree of human genetic homogeneity expected between individuals in small isolated villages may also increase susceptibility to viral infections ${ }^{40}$.

Among the members of the family Caliciviridae detected was the Tulane virus classified in the recovirus genus and originally isolated from a rhesus macaque in a US primate center ${ }^{25,41}$ and subsequently from 5/1614 human diarrhea sample from Bangla-

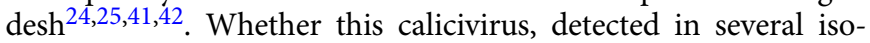
lated village $\mathrm{C}$ children, originated from a human or animal source is unknown but the closer identity of its polyprotein to the human Bangladeshi cases (87\%) versus the Tulane strain from rhesus macaques (52\%) support a likely human source. The single report of this recovirus in a few human diarrhea samples indicates it is a rare human infection ${ }^{24}$, whose transmission from macaque to human is supported by neutralizing antibodies found in a large fraction of non-human primate caretakers ${ }^{43}$.

Another major difference between the enteric human viromes of isolated villages and the large urban center was their higher level of RNA and CRESS DNA eukaryotic viral genomes of unknown origin. Multiple genetic clusters of closely related RNA genomes in the Picornavirales were detected particularly in the most isolated village D (Fig. 4). The genomes are related to those previously described in fecal samples from pigs (posaviruses) ${ }^{44-49,51}$, human (husavirus) $47,50,52$, cat (fesavirus) ${ }^{31}$, fish (fisavirus) $^{53}$, giant panda (pansavirus) ${ }^{54}$, and in a presumably infected Ascaris suum nematode (the large roundworm of pigs) $(\mathrm{JI} 165223)^{55}$. This diverse group of related genomes may reflect viruses shed by nematodes in their gut ${ }^{44}$, a possibility further reinforced by the detection of nematode DNA in the feces of village $\mathrm{B}, \mathrm{C}, \mathrm{D}$ children, or may originate from shared foodstuff from their diet consisting of locally grown plants and hunted preys. Lastly human infections by these genomes cannot be excluded although nucleotide composition of their genomes indicates a likely non-vertebrate source ${ }^{28,47}$.

The cellular hosts of the CRESS-DNA genomes found mainly in village B-D remains similarly uncertain. This rapidly expanding and highly diverse group of viral genomes have been characterized from numerous human and animals feces as well as environmental samples ${ }^{56-62}$. While a few clades of CRESS-DNA have clearly vertebrate (Circoviridae) or plant hosts (Geminiviridae) most CRESS-DNA remain without an identified cellular source ${ }^{62}$. The detection of CRESS-DNA genomes in these human fecal samples may therefore similarly reflect dietary intake from infected foodstuff, infected parasites such as nematodes and protozoan in their gut, or even conceivably their replication in human gut cells. The protozoan and nematode DNA detected in the feces from the isolated villages indicates a higher parasitic burden in these populations than in urban children. High levels of intestinal parasites have been reported in indigenous populations of the Amazon ${ }^{63,64}$. Further studies will be required to determine whether these or other intestinal parasites are the cellular sources of some CRESS-DNA or Picornavirales genomes described here.

As for all similarity-based metagenomics study only viral sequence with protein sequence similarity to any of the currently known viral families could be identified. Therefore sequences from hypothetical human-infecting viral families with no detectable similarity to any viral family in GenBank would not have been recognized.

Unexpectedly, given their isolation, the number of distinct human enteric viruses was not reduced in children from these small, relatively isolated, indigenous villages relative to those from a large urban center. The high incidence and diversity of viral infections in these villages indicates that their relationship to the global enteric virome is not reflective of their physical and cultural isolation. Whether small and presumably even more isolated pre-historic communities (prior to the development of large indigenous population centers) also shed diverse human enteric viruses is unknown. The close genetic similarity of the picornaviruses and caliciviruses strains circulating in the isolated villages to more cosmopolitan viruses support recent viral introductions (Figs. 2 and 3). Such introductions could result from occasional contacts with member of other communities and/or drinking contaminated water. Whether the duration and level of viral shedding or pathogenicity of these enteric infections are greater in children from isolated villages than in urban centers is unknown. Higher susceptibility to viral infections, prolonged and higher level of shedding, and associated diseases could conceivably result from reduced general immunity due to under nourishment and limited medical care. Co-infections with protozoa and nematodes may also influence susceptibility or clearance rate of enteric viral infections and facilitate their persistence in small communities.

It remains to be determined if the high rate and diversity of enteric viral infections detected here are atypical or also occurs in similarly isolated villages from other geographic regions, in even 
more isolated populations such as Yanomami Amerindians ${ }^{65}$, and also applies to their blood and respiratory viromes.

\section{Methods}

Samples selection. Feces samples from apparently healthy children with no recorded symptoms from four different sites in Venezuela were collected in 2015. These locations were different neighborhoods in the capital Caracas (with a population $>4$ million inhabitants) (urban site A) and three Amerindian communities inaccessible by land in the Alto Caura basin, located in Bolivar State in the South of Venezuela, near the border with Brazil (Supplementary Figure 1). Site B, the village of Kanarakuni (GPS: N $4^{\circ} 25^{\prime} 47.4 \mathrm{~W} 64^{\circ} 07^{\prime} 42.9$ ), is a community of the Yekwana indigeneous people, which has $\sim 100$ inhabitants and has some exposure to non-Amerindians (mestizo health and military personnel, since they have a landing strip) (Supplementary Figure 2). The Sanema indigeneous people of site C and $\mathrm{D}$, each with $\sim 50$ inhabitants, are even more remote. Site $\mathrm{C}$ is the village of Surukuma (GPS: $\mathrm{N}^{\circ} 23^{\prime} 8.2 \mathrm{~W}^{\circ} 4^{\circ} 6^{\prime} 45.1$ ) (Supplementary Figure 3) located downstream of village B. Village D, the village of Chajuraña (GPS: N4 ${ }^{\circ} 17^{\prime} 5.5 \mathrm{~W} 64^{\circ}$ $00^{\prime} 41.7$ ) is on a different tributary with no upstream communities and is considered to be even more isolated from outside contact than village C (Supplementary Figure 4). The locations of villages B, C, D, are shown in Supplementary Figure 5. Villages B-C, C-D, and B-D are separated by approximately 4,10 , and 14 straightline miles. One author (O.N.-A.) travels to the villages four times a year to treat onchocerciasis. Two to three tourists also visit the area each year, staying for 1-2 days. Some travel also occurs between villages particularly of the same ethnicity (villages $\mathrm{C}$ and $\mathrm{D}$ ). These sites are distinct from the Yanomami indigenous people of the Upper Orinoco River with apparently no prior recorded contacts with Western people and unusually diverse fecal bacteria populations ${ }^{65}$.

Fecal samples were collected by parents, placed in cryotubes within $2 \mathrm{~h}$ of collection and frozen in liquid nitrogen until analyses. The current study analyzed stool sample from the 20 youngest sampled subjects from each sites totaling 80 samples. Informed consent was obtained from the parent or guardian of each child included in the study. The study was performed following the ethical regulations and was approved by the IRB permit Dir0229/10 from Instituto Venezolano de Investigaciones Científicas (IVIC).

Viral nucleic acid isolation and sequencing. Stool samples were homogenized by vortexing with $1 \mathrm{~mm}$ silica beads in $600 \mu \mathrm{L}$ of phosphate-buffered saline. The homogenate were centrifuged at 12,000 rpm for $5 \mathrm{~min}$ and the fecal supernatant was filtered through a $0.45 \mu \mathrm{m}$ filter (Merck Millipore, Massachusetts, USA) to remove large cell-sized particles. The filtrate was incubated with DNase and RNase enzymes (Turbo DNase, Thermo Fisher Scientific, Massachusetts, USA; Baseline Zero DNase, Epicentre, Wisconsin, USA; Benzonase Nuclease, Novagen, Massachusetts, USA; and RNase A, Thermo Fisher Scientific) at $37^{\circ} \mathrm{C}$ for $90 \mathrm{~min}$ to degrade unprotected nucleic acid ${ }^{35}$. Capsid protected viral nucleic acid were extracted with Agencourt RNAdvance Tissue kit (Beckman Coulter, California, USA) and RiboLock RNase Inhibitor (Thermo Fisher Scientific, Massachusetts, USA) was added to prevent RNA degradation. Reverse transcription was performed with SuperScript III reverse transcriptase (Thermo Fisher Scientific, Massachusetts, USA) using a primer with a random nonamer at the 3 ' end (5'GCCGACTAATGCGTAGTCNNNNNNNNN) and the second strand DNA was synthetized using Klenow Fragment (New England Biolabs, Massachusetts, USA) ${ }^{66}$. The cDNA and DNA was then amplified by PCR with 15 cycles using a primer with the fixed portion of the previous primer (5'GCCGACTAATGCGTAGTC) and AmpliTaq Gold DNA polymerase (Thermo Fisher Scientific, Massachusetts, USA ${ }^{66}$. The amplification products were used to prepare the libraries individually for each sample using the Nextera XT DNA Sample Preparation Kit (Illumina, California, USA) following the manufacturer's instructions, with 15 cycles of PCR amplification ${ }^{67}$ and dual index barcoding. Libraries were sequenced using the Miseq Illumina platform (Illumina, California, USA) in two runs of 40 samples each using 250 bases paired ends sequencing. Each MiSeq run contained ten samples of each group (A, B, C, and D) and libraries from groups A and B shared barcodes between them, but do not shared barcodes with samples from groups $\mathrm{C}$ and $\mathrm{D}$, and vice-versa. To prevent possible batch effects samples were handled in the following order: one fecal sample from site A, then B, then $\mathrm{C}$, then $\mathrm{D}$, then repeat until all samples processed followed by Nextera products pooling for sequencing.

Virome data analysis. Raw reads were pre-processed as described ${ }^{66}$. Bacterial and human host reads were subtracted using Bowtie $2^{68}$ to map the sequences to all bacterial RefSeq genomes and human reference genome hg19. Read bases with Phred quality score lower than ten were trimmed and only one random copy of duplicate reads was retained. The remaining reads were de novo assembled separately for each individual sample using Ensemble Assembler $1.0^{69}$. The contigs generated and single reads to eukaryotic viral sequences from the RefSeq virus protein database using BLASTx. The sequences with significant hits $(E$ value $<$ 0.001 ) were then compared to a non-virus proteome database obtained from GenBank non-redundant database. Sequences hits with lower $E$ value to viruses than to non-viral proteins were classified in their respective viral family and genus. To further extend the contigs generated by de novo assembly using Ensemble
Assembler ${ }^{69}$, the program Geneious ${ }^{\circledR} 9.1$ (Biomatters, EUA) was used to identify reads with sequence similarity to the contig extremities in an iterative fashion.

To quantify the number of reads to different viruses they were matched to viral contigs/genomes using Bowtie $2^{68}$ and reads with $>30$ bp perfect contiguous matches were counted.

We observed samples that were sequenced on the same flow cell (using distinct dual barcodes) as a high viral read sample occasionally contained a small number of reads from the same virus therefore potentially representing false positives. This phenomenon of bleed-over (also known as index spreading or barcode hopping) has been described ${ }^{70-73}$. Bleed-over of up to $0.4 \%$ of the frequency found in the sample with the highest fraction of these viral reads were detected. For example a sample yielding $10^{6}$ reads of which $1 \%$ match a particular virus could be expected to bleed over as many as $\left(10^{6} \times 0.01\right) \times 0.004=40$ reads into another sample generating $10^{6}$ reads. That threshold was used to exclude potential false positives.

Phylogenetic analysis. The open reading frames (ORF) from all complete or partial genomes found were identified and the protein sequences were translated and aligned with Muscle implemented in Geneious ${ }^{\oplus} 9.1$ (Biomatters, EUA). Maximum likelihood phylogenetic tree was generated with PhyML v.3.0 $0^{74}$ using the substitution model determined with Model Generator ${ }^{75}$.

Statistical analysis. The number of reads that matched to each virus was expressed per million total reads and log transformed for statistical analysis. We used Tukey's range test to compare the number of human viruses found per child from each site. Chi square test was performed to compare the proportion of human and non-human viruses detected in each site. Statistical analyses were performed using SPSS (version 20).

\section{Data availability}

Complete or partial genome sequences generated in this study were deposited in GenBank under the accession numbers MG571777 to MG571978. Raw reads were deposited in Sequence Read Archive (SRA) under accession numbers SRR6287056 to SRR6287135.

Received: 13 March 2018 Accepted: 6 September 2018

Published online: 15 October 2018

\section{References}

1. Bonatto, S. L. \& Salzano, F. M. Diversity and age of the four major mtDNA haplogroups, and their implications for the peopling of the New World. Am. J. Hum. Genet. 61, 1413-1423 (1997).

2. Goebel, T., Waters, M. R. \& O'Rourke, D. H. The late Pleistocene dispersal of modern humans in the Americas. Science 319, 1497-1502 (2008).

3. Gilbert, M. T. et al. DNA from pre-Clovis human coprolites in Oregon, North America. Science 320, 786-789 (2008).

4. Dillehay, T. D. et al. Monte Verde: seaweed, food, medicine, and the peopling of South America. Science 320, 784-786 (2008).

5. Dillehay, T. D. et al. New archaeological evidence for an early human presence at Monte Verde, Chile. PLoS ONE 10, e0141923 (2015).

6. Hey, J. On the number of New World founders: a population genetic portrait of the peopling of the Americas. PLoS Biol. 3, e193 (2005).

7. Lell, J. T. et al. The dual origin and Siberian affinities of Native American Y chromosomes. Am. J. Hum. Genet. 70, 192-206 (2002).

8. Raghavan, M. et al. Upper Palaeolithic Siberian genome reveals dual ancestry of Native Americans. Nature 505, 87-91 (2014).

9. Silva, W. A. Jr. et al. Mitochondrial genome diversity of Native Americans supports a single early entry of founder populations into America. Am. J. Hum. Genet. 71, 187-192 (2002).

10. Raghavan, M. et al. POPULATION GENETICS. Genomic evidence for the Pleistocene and recent population history of Native Americans. Science 349, aab3884 (2015).

11. Ishak, R., Machado, L. F. A., Cayres-Vallinoto, I., Guimaraes Ishak, M. O. \& Vallinoto, A. C. R. Infectious agents as markers of human migration toward the Amazon region of Brazil. Front. Microbiol. 8, 1663 (2017).

12. Slattery, J. P., Franchini, G. \& Gessain, A. Genomic evolution, patterns of global dissemination, and interspecies transmission of human and simian T-cell leukemia/lymphotropic viruses. Genome Res. 9, 525-540 (1999).

13. Cayres-Vallinoto, I. M. et al. Human JCV infections as a bio-anthropological marker of the formation of Brazilian Amazonian populations. PLoS ONE 7, e46523 (2012)

14. O'Fallon, B. D. \& Fehren-Schmitz, L. Native Americans experienced a strong population bottleneck coincident with European contact. Proc. Natl Acad. Sci. USA 108, 20444-20448 (2011). 
15. Walker, R. S., Sattenspiel, L. \& Hill, K. R. Mortality from contact-related epidemics among indigenous populations in Greater Amazonia. Sci. Rep. 5 14032 (2015)

16. Black, F. L. Infectious diseases in primitive societies. Science 187, 515-518 (1975).

17. Kapusinszky, U. M. et al. Local virus extinctions following a host population bottleneck. J. Virol. 89, 8152-8161 (2015).

18. Benschop, K., Thomas, X., Serpenti, C., Molenkamp, R. \& Wolthers, K. High prevalence of human Parechovirus $(\mathrm{HPeV})$ genotypes in the Amsterdam region and identification of specific $\mathrm{HPeV}$ variants by direct genotyping of stool samples. J. Clin. Microbiol. 46, 3965-3970 (2008).

19. Graul, S. et al. High diversity of human parechovirus including novel types in stool samples from Ghanaian children. J. Clin. Virol. 96, 116-119 (2017).

20. Kapusinszky, B., Phan, T. G., Kapoor, A. \& Delwart, E. Genetic diversity of the genus Cosavirus in the family Picornaviridae: a new species, recombination, and 26 new genotypes. PLoS ONE 7, e36685 (2012).

21. Holtz, L. R., Finkbeiner, S. R., Kirkwood, C. D. \& Wang, D. Identification of a novel picornavirus related to cosaviruses in a child with acute diarrhea. Virol. J. 5, 159 (2008).

22. Ng, T. F. et al. High variety of known and new RNA and DNA viruses of diverse origins in untreated sewage. J. Virol. 86, 12161-12175 (2012).

23. Yu, J. M., Ao, Y. Y., Liu, N., Li, L. L. \& Duan, Z. J. Salivirus in children and its association with childhood acute gastroenteritis: a paired case-control study. PLoS ONE 10, e0130977 (2015).

24. Smits, S. L. et al. Calicivirus from novel Recovirus genogroup in human diarrhea, Bangladesh. Emerg. Infect. Dis. 18, 1192-1195 (2012).

25. Farkas, T., Sestak, K., Wei, C. \& Jiang, X. Characterization of a rhesus monkey calicivirus representing a new genus of Caliciviridae. J. Virol. 82, 5408-5416 (2008).

26. Chu, D. K. et al. Characterization of a novel gyrovirus in human stool and chicken meat. J. Clin. Virol. 55, 209-213 (2012).

27. Rosario, K. et al. Revisiting the taxonomy of the family Circoviridae: establishment of the genus Cyclovirus and removal of the genus Gyrovirus. Arch. Virol. 162, 1447-1463 (2017)

28. Kapoor, A., Simmonds, P., Lipkin, W. I., Zaidi, S. \& Delwart, E. Use of nucleotide composition analysis to infer hosts for three novel picorna-like viruses. J. Virol. 84, 10322-10328 (2010)

29. Sasaki, M. et al. Metagenomic analysis of the shrew enteric virome reveals novel viruses related to human stool-associated viruses. J. Gen. Virol. 96, 440-452 (2015).

30. Firth, C. et al. Detection of zoonotic pathogens and characterization of novel viruses carried by commensal Rattus norvegicus in New York City. mBio 5, e01933-01914 (2014).

31. Zhang, W. et al. Faecal virome of cats in an animal shelter. J. Gen. Virol. 95, 2553-2564 (2014).

32. Yinda, C. K. et al. Highly diverse population of Picornaviridae and other members of the Picornavirales, in Cameroonian fruit bats. BMC Genom. 18, 249 (2017)

33. Holtz, L. R. et al. Geographic variation in the eukaryotic virome of human diarrhea. Virology 468-470, 556-564 (2014).

34. Kapoor, A. et al. A highly prevalent and genetically diversified Picornaviridae genus in South Asian children. Proc. Natl Acad. Sci. USA 105, 20482-20487 (2008).

35. Victoria, J. G. et al. Metagenomic analyses of viruses in stool samples from children with acute flaccid paralysis. J. Virol. 83, 4642-4651 (2009).

36. Phan, T. G. et al. Acute diarrhea in West African children: diverse enteric viruses and a novel parvovirus genus. J. Virol. 86, 11024-11030 (2012).

37. Linsuwanon, $P$. et al. The fecal virome of children with hand, foot, and mouth disease that tested PCR negative for pathogenic enteroviruses. PLOS ONE 10, e0135573 (2015).

38. Altan, E. et al. Enteric virome of Ethiopian children participating in a clean water intervention trial. PLoS ONE 13, e0202054 (2018).

39. Wang, C. et al. Comprehensive virome analysis reveals the complexity and diversity of the viral spectrum in pediatric patients diagnosed with severe and mild hand-foot-and-mouth disease. Virology 518, 116-125 (2018).

40. King, K. C. \& Lively, C. M. Does genetic diversity limit disease spread in natural host populations? Heredity 109, 199-203 (2012).

41. Handley, S. A. et al. Pathogenic simian immunodeficiency virus infection is associated with expansion of the enteric virome. Cell 151, 253-266 (2012).

42. Farkas, T., Lun, C. W. \& Fey, B. Relationship between genotypes and serotypes of genogroup 1 recoviruses: a model for human norovirus antigenic diversity. J. Gen. Virol. 95, 1469-1478 (2014).

43. Farkas, T. et al. Genetic diversity and histo-blood group antigen interactions of rhesus enteric caliciviruses. J. Virol. 84, 8617-8625 (2010).

44. Shan, T. et al. The fecal virome of pigs on a high-density farm. J. Virol. 85, 11697-11708 (2011).
45. Hause, B. M., Hesse, R. A. \& Anderson, G. A. Identification of a novel Picornavirales virus distantly related to posavirus in swine feces. Virus Genes 51, 144-147 (2015).

46. Sano, K. et al. Identification of further diversity among posaviruses. Arch. Virol. 161, 3541-3548 (2016).

47. Oude Munnink, B. B. et al. Characterization of Posa and Posa-like virus genomes in fecal samples from humans, pigs, rats, and bats collected from a single location in Vietnam. Virus Evol. 3, vex022 (2017).

48. Hause, B. M., Palinski, R., Hesse, R. \& Anderson, G. Highly diverse posaviruses in swine faeces are aquatic in origin. J. Gen. Virol. 97, 1362-1367 (2016).

49. Amimo, J. O. et al. Metagenomic analysis demonstrates the diversity of the fecal virome in asymptomatic pigs in East Africa. Arch. Virol. 161, 887-897 (2016).

50. Phan, M. V. T. et al. Unbiased whole-genome deep sequencing of human and porcine stool samples reveals circulation of multiple groups of rotaviruses and a putative zoonotic infection. Virus Evol. 2, vew027 (2016).

51. Shi, M. et al. Redefining the invertebrate RNA virosphere. Nature 540, 529-543 (2016).

52. Oude Munnink, B. B. et al. A novel genus in the order Picornavirales detected in human stool. J. Gen. Virol. 96, 3440-3443 (2015).

53. Reuter, G., Pankovics, P., Delwart, E. \& Boros, A. A novel posavirus-related single-stranded RNA virus from fish (Cyprinus carpio). Arch. Virol. 160, 565-568 (2015).

54. Zhang, W. et al. Virome comparisons in wild-diseased and healthy captive giant pandas. Microbiome 5, 90 (2017).

55. Wang, J. et al. Deep small RNA sequencing from the nematode Ascaris reveals conservation, functional diversification, and novel developmental profiles. Genome Res. 21, 1462-1477 (2011).

56. Delwart, E. \& Li, L. Rapidly expanding genetic diversity and host range of the Circoviridae viral family and other Rep encoding small circular ssDNA genomes. Virus Res. 164, 114-121 (2012).

57. Rosario, K., Duffy, S. \& Breitbart, M. A field guide to eukaryotic circular single-stranded DNA viruses: insights gained from metagenomics. Arch. Virol. 157, 1851-1871 (2012).

58. Dayaram, A. et al. Diverse circular replication-associated protein encoding viruses circulating in invertebrates within a lake ecosystem. Infect. Genet. Evol. 39, 304-316 (2016).

59. Labonté, J. M. \& Suttle, C. A. Previously unknown and highly divergent ssDNA viruses populate the oceans. Isme. J. 7, 2169-2177 (2013).

60. Krupovic, M., Ghabrial, S. A., Jiang, D. \& Varsani, A. Genomoviridae: a new family of widespread single-stranded DNA viruses. Arch. Virol. 161, 2633-2643 (2016)

61. Ng, T. F. F. et al. A diverse group of small circular ssDNA viral genomes in human Human and non-human primate stools. Virus Evol. 1, vev017 (2015).

62. Simmonds, P. et al. Consensus statement: virus taxonomy in the age of metagenomics. Nat. Rev. Microbiol. 15, 161-168 (2017).

63. Coronato Nunes, B. et al. Spatial and molecular epidemiology of Giardia intestinalis deep deep in the Amazon, Brazil. PLoS ONE 11, e0158805 (2016).

64. Overeem, M. M. et al. Recurrent wheezing is associated with intestinal protozoan infections in Warao Amerindian children in Venezuela: a crosssectional survey. Bmc. Infect. Dis. 14, 293 (2014).

65. Clemente, J. C. et al. The microbiome of uncontacted Amerindians. Sci. Adv. 1, e1500183 (2015).

66. Li, L. et al. Comparing viral metagenomics methods using a highly multiplexed human viral pathogens reagent. J. Virol. Methods 213, 139-146 (2015).

67. Faria, N. R. et al. Establishment and cryptic transmission of Zika virus in Brazil and the Americas. Nature 546, 406-410 (2017).

68. Langmead, B. \& Salzberg, S. L. Fast gapped-read alignment with Bowtie 2. Nat. Methods 9, 357-359 (2012)

69. Deng, X. et al. An ensemble strategy that significantly improves de novo assembly of microbial genomes from metagenomic next-generation sequencing data. Nucleic Acids Res. 43, e46 (2015).

70. Yang, J. et al. Unbiased parallel detection of viral pathogens in clinical samples by use of a metagenomic approach. J. Clin. Microbiol. 49, 3463-3469 (2011).

71. Wilson, M. R. et al. Multiplexed metagenomic deep sequencing to analyze the composition of high-priority pathogen reagents. mSystems 1, e00058-16 (2016).

72. Mitra, A., Skrzypczak, M., Ginalski, K. \& Rowicka, M. Strategies for achieving high sequencing accuracy for low diversity samples and avoiding sample bleeding using illumina platform. PLoS ONE 10, e0120520 (2015).

73. Moustafa, A. et al. The blood DNA virome in 8,000 humans. PLoS. Pathog. 13, e1006292 (2017).

74. Guindon, S. et al. New algorithms and methods to estimate maximumlikelihood phylogenies: assessing the performance of PhyML 3.0. Syst. Biol. 59, 307-321 (2010).

75. Keane, T. M., Creevey, C. J., Pentony, M. M., Naughton, T. J. \& McLnerney, J. O. Assessment of methods for amino acid matrix selection and their use on 
empirical data shows that ad hoc assumptions for choice of matrix are not justified. BMC Evol. Biol. 6, 29 (2006).

\section{Acknowledgements}

J.D.S. was recipient of a fellowship by the Brazilian Research Council under the auspices of Science without Borders Program and is supplemented with intramural funds by the Brazilian National Cancer Institute. National Heart, Lung, and Blood Institute R01 HL105770 grant and Blood Systems Research Institute for support to D.X. and E.D.

\section{Author contributions}

J.D.S. generated the libraries, assembled genomes, submitted data to GenBank, performed phylogenetic analyses, and assisted in drafting manuscript; M.G.D.-B., M.C. and O.N.-A. designed the microbiome project; M.G.D.-B., M.C., O.L., H.C. and O.N.-A. collected samples and epidemiological data in the field; D.X. assembled BLASTx, Bowtie2, and de novo assembly pipelines for sequence analyses; E.D. directed the viral study and wrote manuscript. All authors reviewed the manuscript.

\section{Additional information}

Supplementary Information accompanies this paper at https://doi.org/10.1038/s41467018-06502-9.

Competing interests: The authors declare no competing interests.
Reprints and permission information is available online at http://npg.nature.com/ reprintsandpermissions/

Publisher's note: Springer Nature remains neutral with regard to jurisdictional claims in published maps and institutional affiliations.

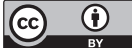

Open Access This article is licensed under a Creative Commons Attribution 4.0 International License, which permits use, sharing, adaptation, distribution and reproduction in any medium or format, as long as you give appropriate credit to the original author(s) and the source, provide a link to the Creative Commons license, and indicate if changes were made. The images or other third party material in this article are included in the article's Creative Commons license, unless indicated otherwise in a credit line to the material. If material is not included in the article's Creative Commons license and your intended use is not permitted by statutory regulation or exceeds the permitted use, you will need to obtain permission directly from the copyright holder. To view a copy of this license, visit http://creativecommons.org/ licenses/by/4.0/.

(C) The Author(s) 2018 